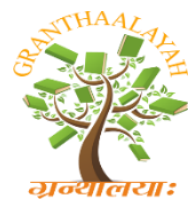

\author{
INTERNATIONAL JOURNAL OF RESEARCH - \\ GRANTHAALAYAH \\ A knowledge Repository
}

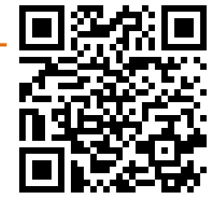

Science

\title{
ARTIFICIAL INTELLIGENCE AND ITS APPLICATION IN PHARMACEUTICAL MEDICINES
}

\author{
Dr. Pramod Kumar ${ }^{* 1}$ \\ ${ }^{* 1}$ Research and Development Centre, Akums Drugs and Pharmaceuticals Pvt. Ltd Haridwar
}

Uttarakhand, India

\begin{abstract}
Big data in the life sciences and healthcare sectors is an increasing trend. It is the processing and displaying of huge volumes and varieties of data at a rapid speed. There is a vast amount of data in the healthcare and Pharmaceutical industries, including lab data, insurance data, patient records, research data, and even social media data $(1,2)$. Pharmaceutical companies have vast amounts of compounds that could be the perfect solution to combat specific diseases, but they have no way to identify them as such. The development and production of drugs can cost pharmaceutical companies up to $\$ 2.6$ billion (£1.8bn) and take 12 to 14 years to complete (1). Artificial Intelligence (AI) plays a crucial role in enabling the industry to achieve these objectives, be it analytics in personalized medicine, cloud computing in collaboration, or wearable devices in remote and self-health monitoring. Thus, the main short/medium-term implication AI has for the pharmaceutical industry is the reduced time it takes to develop drugs and thus the associated costs, enhancing return on investment and could even mean a reduction in cost for end users. As the pharmaceutical industry becomes increasingly more connected, information and communication technologies will fundamentally reshape both the consumption and delivery of medications (1, $2,3)$.
\end{abstract}

Keywords: Artificial; Pharmaceutical; Application.

Cite This Article: Dr. Pramod Kumar. (2019). "ARTIFICIAL INTELLIGENCE AND ITS APPLICATION IN PHARMACEUTICAL MEDICINES." International Journal of Research - Granthaalayah, 7(9), 382-385. https://doi.org/10.29121/granthaalayah.v7.i9.2019.623.

\section{Introduction}

\subsection{Application of AI in Pharmaceuticals}

\subsubsection{Research and Development $(1,2,3)$}

\section{BERG}

A Boston based Pharma start up, BERG uses a data driven approach to drug discovery. This is the company where math meets biology, and the firm believes this combination is key to discovering 
the correct drugs for complex diseases. Their software uses AI to process huge amounts of biological data to reveal what happens (in minute detail) in the journey from good health to cancer. This allows better insights, more informed hypothesis, and thus more efficient drug development. The AI program processes a huge amount of data. Aside from the genomic information, one single cell tissue could produce over 14 trillion data points. Using this approach, the team discovered the key role mitochondria had in allowing cancer cells to flourish in pancreatic cancer. Cancer cells can turn off the mitochondria which results in them losing their ability to die. BERG's drug BPM 31510 helps the mitochondria function and essentially turns the cancer cells back into normal cells. The team believes they can use this model for other forms of cancer.

\section{Insilico Medicine}

This bioinformatics firm has taught its AI system to predict the therapeutic use of new drugs before they even enter the testing process. The AI processes huge amounts of data from experiments on human cells using known drugs. Over time, the AI program was able to achieve roughly 55\% accuracy identifying one out of 12 of the drugs therapeutic applications. Although this seems like a small number, it is actually a big step for researchers who would have to do many experiments (both time consuming and costly) to reach the same conclusions.

\section{Houston Methodist Research Institute}

Researchers at Houston Methodist Research Institute have developed an AI model that can predict breast cancer risk, allowing doctors to closely monitor those at future risk. Currently, around half of mammograms yield false results, according to the American Cancer Society, and means evasive procedures are sometimes unnecessary. The program interprets mammograms and translates patient data into diagnostic information 30 times faster than a doctor, and with $99 \%$ accuracy. Manual review of 50 charts took two clinicians 50 to 70 hours, whereas the AI software reviewed 500 charts in a few hours, saving the human doctors 500 hours of their time. It scans patient charts, collects diagnostic features and correlates mammogram findings with breast cancer subtypes. With this information, clinicians used the results to accurately predict the patient's probability of breast cancer diagnosis.

\section{Eve}

Through machine learning and hypothesis testing, The University of Manchester's 'robotic scientist' Eve recognises why it has succeeded and thus improves at the tasks it needs to perform. Eve is designed to automate early stage drug development and has already discovered lead compounds against malaria and African sleeping sickness.

\subsubsection{Commercial Effectiveness $(2,3,4)$}

Artificial Intelligence offers actionable insights generated from real-world data to provide greater predictive intelligence relating to patients and expected outcomes. By incorporating numerous care factors including patient's health history, genetic disposition, lifestyle indicators and environmental factors - Pharmaceuticals are getting closer and closer to finally offering personalized, precision medicines. Through target audience marketing, pharmaceutical companies can further assure the right information is presented at the right time to facilitate informed patient and provider discussions. 


\subsubsection{Pharmacovigilance $(2,3,4)$}

Pharmaceutical companies rely on many data points to ensure the safety of their products. Clinical trials track the effectiveness of a particular medication. In addition, they track any adverse effects experienced by participants. Further, healthcare providers must track interactions and complications when multiple medications are prescribed for treatment. This may change over time, as patients may develop resistance to certain medications or as more medications are introduced to combat side effects experienced from certain treatment options. Further complicating the process, certain patients may develop dependencies on medications like painkillers or not reveal all of the medications being consumed. Patients who seek to abuse certain medications may seek to source them from different providers. Connected Pharma will be able to access real-time prescription data to flag abuses and possible dangerous interactions.

\section{Limitations of Artificial Intelligence (5)}

1) High Cost: Creation of smart technologies can be expensive, due to their complex nature and the need for repair and ongoing maintenance

2) Soft ware up gradation/ Maintenance: Software programs need regular upgrading to adapt to the changing business environment and, in case of breakdown, present a risk of losing code or important data. Restoring this is often time-consuming and costly.

3) Implementation Time: Implementation times of the system which are often lengthy.

4) Integration challenges of software and lack of knowledge of the state-of-the-art systems.

5) Usability and interoperability with other systems and platforms.

\subsection{Regulatory bodies stand regarding AI use $(4,6)$}

The FDA has been leading initiatives with the pharmaceutical, biological, and medical device industries to try to increase the ownership of product quality to the industry through the Center for Devices and Radiological Health (CDRH) "Case for Quality", identify proactive signals of risk to product quality through the Center for Drug Evaluation and Research (CDER) "Call for Metrics", reduce the risk of drug shortages, and utilize more holistic information during product approval that would allow FDA to grant a "pre-certification" review process that is similar to the Transportation Security Administration (TSA) pre-check. Artificial intelligence can increase the dataset of relevant internal and external information to support these and other goals of the FDA. As a result, several FDA officials are noting the power of AI but, as with industry, are exploring the possibilities, obstacles, and areas that are still not quite understood. FDA's Bakul Patel, for example, is exploring the power of artificial intelligence in the newly formed Digital Health Unit that reports directly to the CDRH center director, Jeff Shuren.

Through the Xavier Artificial Intelligence Initiative, FDA and industry members will form a team to explore how AI can be used to continuously assess product quality, which could enable industry to move from the proactive measures afforded by metrics to the predictive capabilities of artificial intelligence. 


\section{Future Prospects $(1,7)$}

Processing large clinical and medical data isn't the only place where AI could affect the Pharma industry. Even business and marketing based decisions could be helped by computing 'brains', for example by analyzing and assisting with mergers and acquisitions and providing guidance on the most efficient and effect way to market new products.

Although AI is in its infancy, the fusion of math's, technology, biology and healthcare is becoming more and more talked about in the industry and progress in being made in developing systems with real-world value. There is wider talk of AI being an existential threat to humanity (Stephen Hawking, Bill Gates and Elon Musk have all voiced their words of warning), but in the specific application of pharmaceuticals and treating diseases, then any increase in efficiency and effectiveness can only be welcomed.

\section{References}

[1] How-artificial-intelligence-is-being-used-in-the-pharmaceutical-industry https://www.nesgt.com/blog/2016/10:

[2] What to expect from artificial intelligence in Pharma and how to get there https://www.pharmaceuticalonline.com

[3] Artificial-intelligence-drug-discovery: https://www.drugtargetreview.com/article/15400.

[4] Understanding the potential of artificial intelligence across pharmaceutical lifecycle: http://www.pharmtech.com

[5] Advantages and disadvantages of artificial-intelligence: https://content.wisestep.com.

[6] ComputerWorld, "With IBM's Watson, GlaxoSmithKline tackles sniffle and cough question," October 24, 2016.

[7] ARTIFICIAL INTELLIGENCE: NEXT FRONTIER FOR CONNECTED PHARMA: www.scalablehealth.com.

*Corresponding author.

E-mail address: pramod_79kumar@ rediffmail.com 